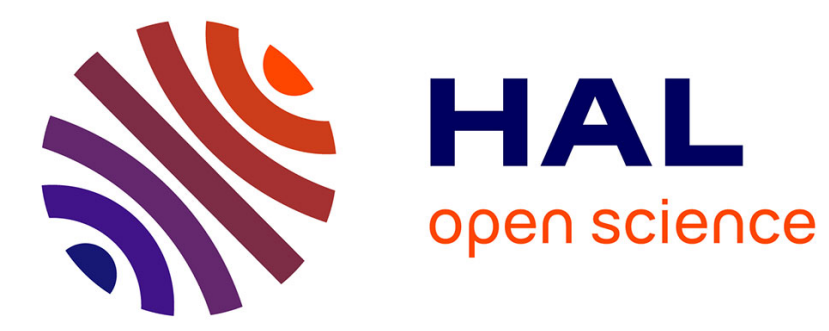

\title{
Visual quality of printed surfaces: Study of homogeneity
}

David Nébouy, Mathieu Hébert, Thierry Fournel, Jean-Luc Lesur

\section{To cite this version:}

David Nébouy, Mathieu Hébert, Thierry Fournel, Jean-Luc Lesur. Visual quality of printed surfaces: Study of homogeneity. Proceedings of SPIE volume 9016, Image Quality and System Performance XI, 2014, pp.9016-12. hal-00962259

\section{HAL Id: hal-00962259 \\ https://hal.science/hal-00962259}

Submitted on 25 Mar 2014

HAL is a multi-disciplinary open access archive for the deposit and dissemination of scientific research documents, whether they are published or not. The documents may come from teaching and research institutions in France or abroad, or from public or private research centers.
L'archive ouverte pluridisciplinaire HAL, est destinée au dépôt et à la diffusion de documents scientifiques de niveau recherche, publiés ou non, émanant des établissements d'enseignement et de recherche français ou étrangers, des laboratoires publics ou privés. 


\title{
Visual quality of printed surfaces: Study of homogeneity
}

\author{
D. Nébouy ${ }^{\mathrm{a}, \mathrm{b}}$, M. Hébert ${ }^{\mathrm{a}}$, T. Fournel ${ }^{\mathrm{a}}$, J.-L. Lesur $^{\mathrm{b}}$ \\ ${ }^{a}$ Université de Lyon, Université Jean Monnet de Saint Etienne, CNRS UMR 5516, Laboratoire \\ Hubert Curien, F- 42000 Saint Etienne, FRANCE; ${ }^{\mathrm{b}}$ Gemalto SA Avenue du pic de Bertagne, ZA de \\ Gémenos, BP.100 F-13881 Gémenos Cedex, France.
}

\begin{abstract}
This paper introduces a homogeneity assessment method for the printed versions of uniform color images. This parameter has been specifically selected as one of the relevant attributes of printing quality. The method relies on image processing algorithms from a scanned image of the printed surface, especially the computation of gray level cooccurrence matrices and of objective homogeneity attribute inspired of Haralick's parameters. The viewing distance is also taken into account when computing the homogeneity index. Resizing and filtering of the scanned image are performed in order to keep the level of details visible by a standard human observer at short and long distances. The combination of the obtained homogeneity scores on both high and low resolution images provides a homogeneity index, which can be computed for any printed version of a uniform digital image. We tested the method on several hardcopies of a same image, and compared the scores to the empirical evaluations carried out by non-expert observers who were asked to sort the samples and to place them on a metric scale. Our experiments show a good matching between the sorting by the observers and the score computed by our algorithm.
\end{abstract}

Keywords: Print quality assessment, Halftoning, Quality index, Quality attributes, Uniformity, Gray-level co-occurrence matrix, Human Visual System, Appearance, Scanner, Printing technologies

\section{INTRODUCTION}

Printing has evolved significantly the last few years, due to first a diversification of processes, and also to the digital revolution [1] including an automation of the processes without the expertise of the printman at the printing time. With this rapid evolution, the quality of color images reproduced on hard supports has considerably increased, and the amazing diversification of these techniques permitted by the new digital technologies provides a large panel of solutions for image reproduction. This leads to an issue for comparing the different techniques in terms of quality, an intuitive concept which is generally well appreciable visually but difficult to assess with objective, measurable values. Scientific concern for printing quality is recent: In [2], the printing quality is explored by defining various quality attributes: color, lightness, sharpness, contrast, physical, artifacts. These attributes were estimated from psycho-visual experiments carried out by a panel of 15 observers with various expertise. In contrast with Pedersen et al. [3], [4], we believe that an important distinction should be made between printing quality (related to the degradation of the original digital image when transferred to the paper or plastic support) and image quality (including printing quality plus the perceived quality of the original image). Our opinion is that, contrast, lightness, and color rather qualify the image quality. The present work focuses on the homogeneity of tones i.e. the homogeneity of any surfaces on which is printed an originally uniform digital color image. This attribute is considered as relevant in the standard ISO 13660: 2001, the only international standard describing a wide range of attributes of image quality dedicated to quality assessment for binary or monochrome printing systems. [5]. In this standard, mottling is defined as a type of homogeneity default of paper-like surfaces and an index is suggested in the spatial domain. A pass-band method was proposed [6] as an alternative able to better take into account the sensitivity of the human visual system. A pass-band assessment funded on wavelets, followed by a second-order statistics calculation on the gray-level co-occurrence matrix was achieved in [7] to form an homogeneity index. Our aim here is to integrate human vision data into both the filtering step and the second-order statistics calculation. As in [6], our approach first consists in the digitalization of the printed surface using a high resolution flatbed office scanner, the scanned image being then processed in order to compute an homogeneity index. It then consists in comparing this score for many samples (several versions of a same digital image printed on different supports with different printing techniques) with the empirical evaluation by observers of these samples on a scale. 
In the next sections, we first explain how the surfaces are digitalized and which tool of image processing we use before developing in details our method and its tunable parameters. We finally compare the score provided by the method with empirical assessment by observers on four sets of samples.

\section{DIGITALIZATION OF THE PRINTS}

For the digitalization of the samples, a scanner is used. An initial study on scanners have led to the conclusion that it is a reliable tool for image acquisition due to its remarquable reproductability, mainly performed by a uniform illumination, and good geometry that avoids specular reflections when the surface is flat. However, spatial and color distortions induced by the acquisition systems should be taken into account when assessing quality.

The color gamut of our human visual system is much larger than that of the scanner, which is unknown but can be assumed to be quite close to sRGB. Because of this, the color distortion problem is twofold: First, the so-called "seen by the scanner" colors are displaced within the very gamut of the scanner, but also, it may appears that different printable colors are out of the gamut of the scanner and thus perceived by the scanner as having the same color. A color calibration would seem necessary to solve at least the first issue. However, we noticed that very often after color correction, images are noisier than before correction. As part of our work (the assessment of uniformity), we chose not to apply color correction to avoid the risk of disrupting our measure.

Regarding the spatial distortions, there is a significant horizontal drift. In the vertical direction, we do not notice geometric drift, the motor is properly position controlled. This drift can be corrected by adjusting the position of the pixels by polynomial interpolation or spline on each line of the captured image. But such treatment would create a change in the value of certain pixels on each line, the most affected by this drift being on the edges of each line, and would lead to a loss of the native resolution of the image as well as local color unwanted distortions. It is preferable to analyze the scanner profile derives and locate the sample on the scanner glass in an area where the drifts are minimal.

We recommend scanning samples at a high resolution (e.g. $2400 \mathrm{dpi}$ ), and then reducing to 1200 dpi, the initial resolution of our method, in order to perform controlled down-sampling.

\section{BACKGROUND: IMAGE HOMOGENEITY ANALYSIS}

The printed copy of a uniform color patch can be considered as a texture. In image processing, a classical tool in texture characterization relies on Haralick's parameters derived from the gray-level co-occurrence matrix (GLCM) also called gray-tone spatial-dependence matrix, first presented in [8]. In contrast with first-order statistic tools (histogram, mean, standard deviation, skewness, and kurtosis), the GLCM is a statistic tool of $2^{\text {nd }}$ order [9]. First order statistics are representative of gray-level distribution of the pixels in the image regardless of their spatial arrangement, whereas second-order statistics involve two pixels simultaneously.

The construction of the GLCM $H_{t}$ is defined as:

$$
\mathbf{H}_{\mathbf{t}}=\left(h_{\mathbf{t}}(i, j)\right)_{\left.i \in \llbracket 0.2^{n} b\right], j \in \llbracket 0.2^{n t} \rrbracket}
$$

with

$$
h_{\mathbf{t}}(i, j)=\operatorname{Card}\left\{\{p, p+\mathbf{t}\} \in I^{2} ; f(p)=i \text { and } f(p+\mathbf{t})=j\right\}
$$

where $I$ is a rectangular image encoded on $n_{b}$ bits, $f$ is an application from $I \times I \rightarrow \llbracket 0 . .2^{n_{b}}-1 \rrbracket$, Card means the cardinality of a set, $\mathbf{t}$ is a given translation vector, and $\{p, p+\mathbf{t}\}$ is a pair of pixels in image $I$. The vector $\mathbf{t}$ may be specified by a unit vector $\mathbf{w}$ and a distance $D$ :

$$
\mathbf{t}=D \cdot \mathbf{w}
$$

Starting from an initialization to the null matrix, $\mathbf{H}_{\mathbf{t}}$ is incrementally built by considering in turn every pair of pixels $\{p, p+\mathbf{t}\}$ in image $I$, and incrementing by one the entry $h_{\mathbf{t}}(f(p), f(p+\mathbf{t}))$ (see Figure 1$)$. 
After normalization of this matrix, each entry $h_{\mathbf{t}}(i, j)$ means the probability to transit, along a vector $\mathbf{t}$, from a pixel with value $i$ to a pixel with value $j$ :

$$
p_{\mathbf{t}}(i, j)=\frac{h_{\mathbf{t}}(i, j)}{\operatorname{Card}\left\{I \cap T_{-\mathbf{t}}(I)\right\}}
$$

with $T_{\mathbf{x}}$ means the translation operator of a vector $\mathbf{x}$.

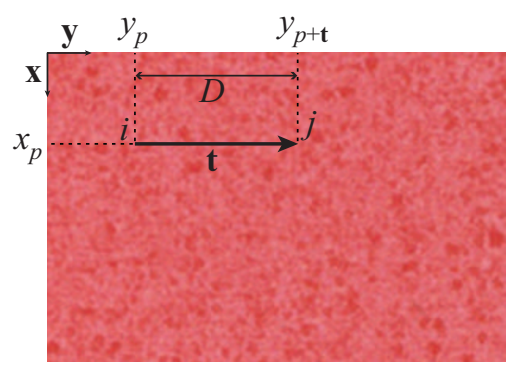

Figure 1. Scanning of the image pixels for every point $\left(x_{p}, y_{p}\right)$ in order to build the gray level co-occurrence matrix.

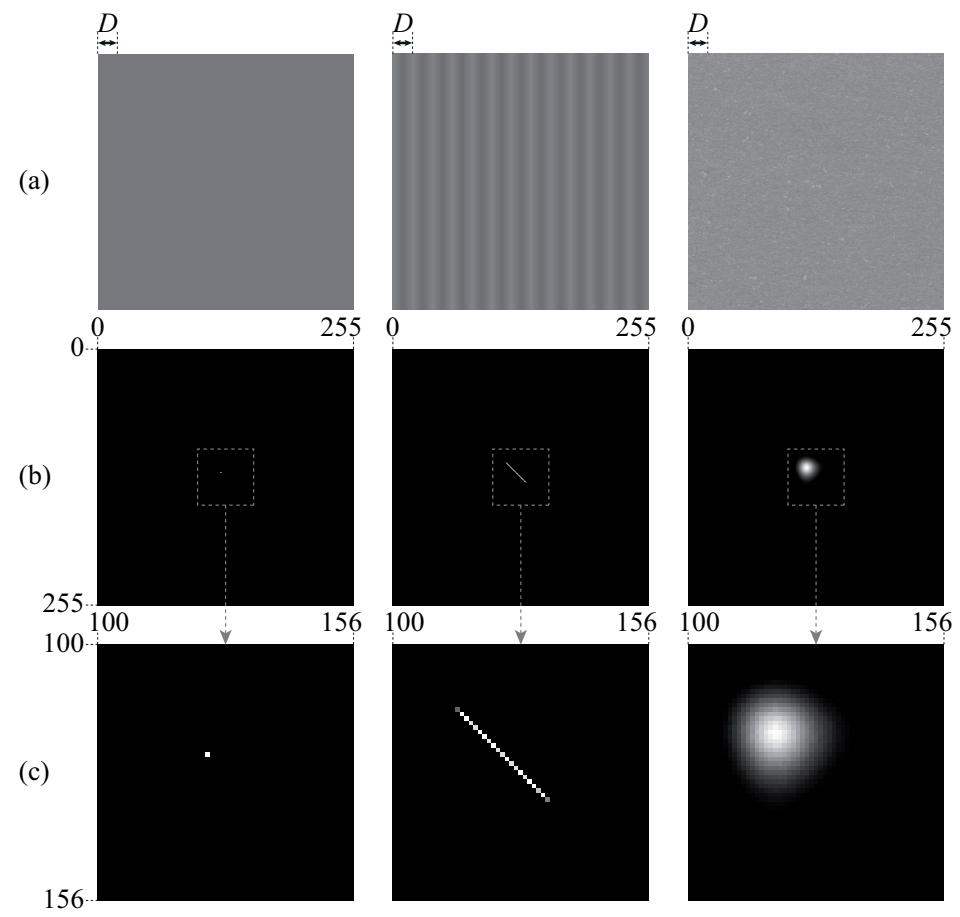

Figure 2. (a) Three original gray level images. (b) Gray level representation of the corresponding GLCM where the white color means the max value. (c) Zoom on the center of images (b).

On such GLCM, Haralick defines several texture attributes among which an homogeneity attribute comprised between 0 and 1, defined as:

$$
H o m=\sum_{i=0}^{2^{n_{b}}} \sum_{j=0}^{2^{n_{b}}} \frac{1}{1+(i-j)^{2}} \times p_{\mathbf{t}}(i, j)
$$


If the GLCM $\mathbf{H}_{\mathbf{t}}$ of image $I$ is computed on a uniform image (top left image on Figure 2) or on a periodical image with period $\mathbf{t}$ (top middle image), then for every pair of pixels $(f(p), f(p+\mathbf{t}))$, we have $f(p)=f(p+\mathbf{t})$. Therefore, only entries in the diagonal of $\mathbf{H}_{\mathbf{t}}$ are incremented; and Hom $=1$. In the other cases (for example for the top right image of Figure 2), $\mathbf{H}_{\mathbf{t}}$ is not a diagonal matrix, and $0<H o m<1$.

Formula (5) may be replaced with other ones, defined so as to give different weight to entries more distant from the diagonal. For example, we may generalize Eq. (5) by defining the following function weighting the transition probability $p_{t}(i, j)$ :

$$
w_{a, b}: x \mapsto \frac{1}{1+a \times x^{b}}
$$

where $a$ and $b$ are tunable real values (see Figure 3), and $x=|i-j|$.

Using $w_{a, b}$ with different $a$ and $b$ values than the ones used in Eq. (5), we can tune the response of the homogeneity score to variations of the pixel values in the image.

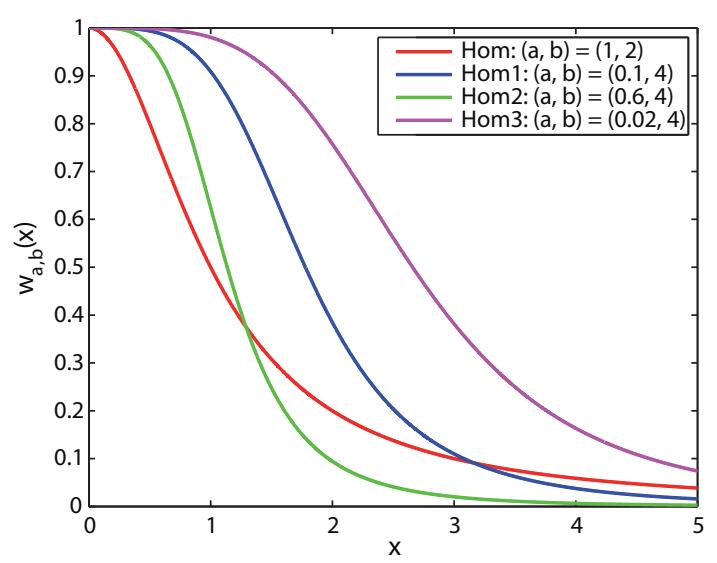

Figure 3. $w_{a, b}$ function for three different sets of $(a, b)$ values. Original Haralick's attribute (denoted as Hom) corresponds to the $w_{1,2}$ function plotted in red line. $w_{0.1,4}$ and $w_{0.6,4}$ functions, respectively denoted as Hom 1 and Hom 2 , are plotted in blue, respectively green lines.

\section{OVERVIEW OF THE METHOD}

The Homogeneity assessment method that we propose relies on the following steps:

- Preprocessing, including image resizing and filtering in order to simulate near vision at High Resolution (HR), and far vision at Low Resolution (LR),

- $\quad$ Application of the Image Homogeneity Analysis method described in Section 3.

- $\quad$ Score computation from the GLCM on both HR and LR images.

\section{PREPROCESSING}

This section intends to modify the raw scanned image into a new one, closer to human perception. A three step preprocessing of the scanned images is performed: Creation of custom color channels from the initial RGB channels; filtering in order to take account of human visual resolution; quantization (optional). 
For the HR image, a 1200 pixel per inch (ppi) image is recommended. A bicubic interpolation (scale of $1 / 2$ if the input image is $2400 \mathrm{ppi}$ ) with antialiasing method is selected to perform image resizing.

The first step is to change the color space. Scanner yields R,G,B values for each pixel. We could process these R,G, and $\mathrm{B}$ channels each ones as a grayscale image, but they do not contain the optimal information. In order to be more consistent with human perception, we prefer converting the RGB values into CIELAB values by considering that the input RGB values are represented in the Adobe RGB (1998) color space, then converting into CIE1931XYZ for a $2^{\circ}$ standard observer, and finally converting in CIELAB by selecting the D65 illuminant. We analyze the $L^{*}$ (lightness) channel, and the $h_{a b}$ (hue) channel. CIELAB color space is chosen because it is a color appearance model [10] (pages $160-161)$. The $a^{*}$ and $b^{*}$ Cartesian coordinates are transformed into cylindrical coordinates $C_{a b}^{*}$ and $h_{a b}$, respectively defined as

$$
\begin{aligned}
& C_{a b}^{*}=\sqrt{a^{* 2}+b^{* 2}} \\
& h_{a b}=\tan ^{-1}\left(\frac{b^{*}}{a^{*}}\right)
\end{aligned}
$$

Implementation remark: $\operatorname{atan}(y / x)$ function yields an angle in $] \frac{-\pi}{2}, \frac{\pi}{2}[$ which is not defined if $x=0$. Alternative function is available in most computation softwares, often denoted as $\operatorname{atan} 2(y, x)$, yielding an angle in $]-\pi, \pi]$, defined for every $y$ and $x$.

Note that it is also possible to define the luminance image directly from RGB values, using the NTSC luminance (Y) formula, first presented in Ref. [11]:

$$
Y=0.299 \times R+0.587 \times G+0.114 \times B
$$

The second step corresponds to the simulation of the blur produced by our human eyes. According to Hermann von Helmholtz's observations [12], we consider the definition of the "normal visual acuity" as the ability to resolve a spatial pattern separated by a visual angle $\alpha$ of one minute of arc. Thus, giving an observation distance $d$ (in $\mathrm{cm}$ ) and a scan resolution of the image $r_{s}$ (in ppi), we can define the convolution kernel of the blurring filter (Figure 4). The size $s$ of the kernel depends on the standard deviation $\sigma$ used for the Gaussian profile:

$$
\begin{gathered}
\sigma=d \times \tan \left(\frac{1}{60}\right) \times \frac{r_{s}}{2.54} \\
s=6 \times \sigma+1
\end{gathered}
$$

We choose to simulate the HR images as viewed at a short distance $(d=15 \mathrm{~cm})$, near the human punctum proximum, which corresponds to $\sigma=2.06$ pixels, i.e. $s=13$.

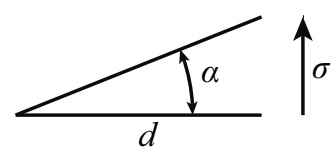

Figure 4. Definition of $\sigma$ knowing the visual angular resolution $\alpha=1^{\prime}=\frac{1}{60}$ degree at the viewing distance $d$.

Lastly, a quantization step (e.g. on $n_{b} \leq 8$ bits for each channel), can eventually be performed, with the consequence to decrease the size of the GLCM but also to lose the least significant bits (i.e. small grey-level variations in the image).

For the low resolution images, we perform the same 3 steps with a 240 ppi resolution image (scale of $1 / 10$ if the input is $2400 \mathrm{ppi}$ ), with $\sigma=1.03$ pixels and $t=7$, which corresponds to the image viewed by an observer at about $40 \mathrm{~cm}$.

The preprocessing step leads to 4 images, denoted as $Y^{(H R)}, Y^{(L R)}, h_{a b}^{(H R)}, h_{a b}^{(L R)}$. 


\section{APPLICATION OF THE IMAGE HOMOGENEITY ANALYSIS METHOD}

We apply the method presented in Section 3 on the four images $Y^{(H R)}, Y^{(L R)}, h_{a b}^{(H R)}, h_{a b}^{(L R)}$ obtained in the preprocessing step.

For each of the two HR images, we choose the following translation vectors $\mathbf{t}_{1}$ and $\mathbf{t}_{\mathbf{2}}$ to compute respectively GLCMs $\mathbf{M}_{1}$ and $\mathbf{M}_{2}$ :

$$
\begin{aligned}
& \mathbf{t}_{\mathbf{1}}=D^{(H R)} \cdot \mathbf{u}_{\mathbf{1}} \\
& \mathbf{t}_{\mathbf{2}}=D^{(H R)} \cdot \mathbf{u}_{\mathbf{2}}
\end{aligned}
$$

with $D^{(H R)}=25$ pixels (corresponding to a physical displacement of about $0.5 \mathrm{~mm}$ on 1200 ppi image), and $\mathbf{u}_{1}=\mathbf{y}$, $\mathbf{u}_{2}=\mathbf{x}$ the horizontal, respectively vertical unit vectors (see Figure 1).

We then compute a GLCM $\mathbf{M}$ as the average of $\mathbf{M}_{1}$ and $\mathbf{M}_{2}$ :

$$
\mathbf{M}=\left(\frac{\mathbf{M}_{1}+\mathbf{M}_{2}}{2}\right)
$$

We also compute a chroma value on the HR image, denoted as $c^{(H R)}$ and defined as the average of the chroma values computed from Eq. (7) on each pixel of the image. From matrix M, we compute the homogeneity attribute Hom 1 according to Eq. (6) with a value of $a$ denoted $a^{(H R)}$ depending on $c^{(H R)}$ and given in Figure 5.a, and $b=4$.

For each of the two LR images, we perform similar processing as above with $D^{(L R)}=35$ pixels (corresponding to a physical displacement of about $0.37 \mathrm{~cm}$ on 240 ppi images), and compute the Hom2 attribute using the $w_{a^{(L R)}, 4}$ function defined by Eq. (6), where $a^{(L R)}$ is given by Figure 5.b as a function of $c^{(L R)}$ computed as $c^{(H R)}$ on the chroma values of the LR image.

These values for $D, a$, and $b$, have been chosen because they seem the most relevant in our study according to physical and empirical considerations.

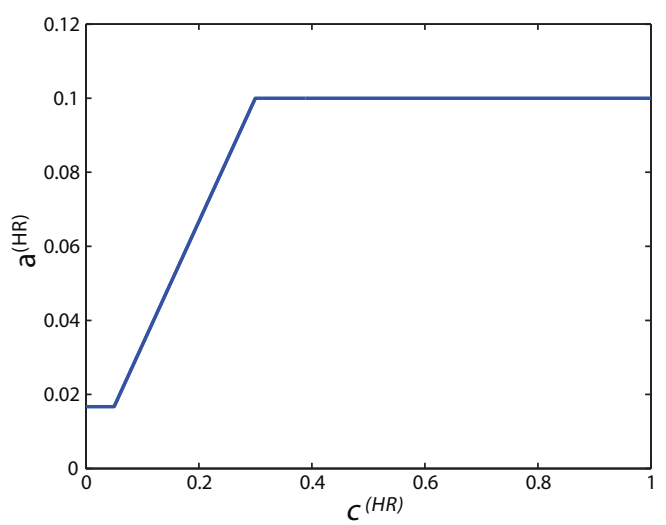

(a)

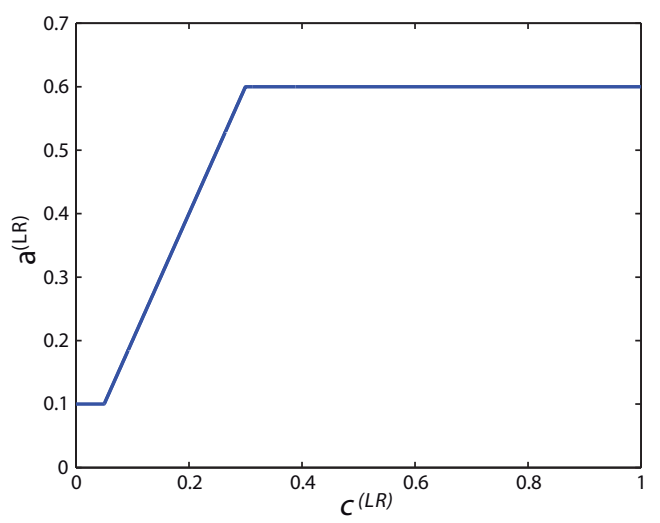

(b)

Figure 5. Variation of the $a^{(H R)}$ and $a^{(L R)}$ values as a function of the chroma values $c^{(H R)}$, respectively $c^{(L R)}$, for HR and LR images. 


\section{CONSTRUCTION OF THE OBJECTIVE SCORE}

The computation of homogeneity attribute on the GLCM of $Y^{(H R)}, Y^{(L R)}, h_{a b}^{(H R)}, h_{a b}^{(L R)}$ yields four values to be combined in order to obtain the final homogeneity score. Let's denote these four values as $H_{Y^{(H R)}}, H_{h_{a b}^{(H R)}}, H_{Y^{(L R)}}, H_{h_{a b}^{(L R)}}$.

Our experience shows that relevant homogeneity score in respect to human perception is given by the following empirical formula:

$$
\min \left(\frac{H_{Y^{(H R)}}+c^{(H R)} \times H_{h_{a b}^{(H R)}}}{1+c^{(H R)}}, \frac{H_{Y^{(L R)}}+c^{(L R)} \times H_{h_{a b}^{(L R)}}}{1+c^{(L R)}}\right)
$$

Notice that the chroma is taken into account in this formula through the parameters $c^{(H R)}$ and $c^{(L R)}$ computed in Section 6 because we noticed that the influence of the homogeneity parameter on the hue image is lower when the colors are not saturated. The weight attributed to the luminance channel is higher than the one attributed to the hue channel $(0 \leq c \leq 1$, and in practice, on prints, it is rather $0 \leq c \leq 0.6)$ as it is commonly done in video compression for digital television video encoding where the luminance channel has a double bandwidth than the chromatic ones (See for example [13]).

\section{REFERENCED PARAMETERS OF THE METHOD}

The referenced parameters of our method are:

- Quantization (images encoded on $n_{b}$ number of bits): The less is quantization, the more careful details are kept. It seems better to keep all the details. Instead of decreasing $n_{b}$ below the usual value of 8 bits, we prefer adjusting the $a$ and $b$ parameters in formula (6).

- Size of blurring kernel: it is set by the considered viewing distance $d$, which is about $15 \mathrm{~cm}$ for near vision, and $40 \mathrm{~cm}$ for far vision.

- Scan resolution: The size of one pixel in HR images is about $21 \mu \mathrm{m}$ after downsampling original 2400 ppi scanned image to 1200 ppi. This appears sufficiently accurate in comparison to the human vision and to the finest commercial printing systems available today.

- $D^{(H R)}$ and $D^{(L R)}$ : They must be representative of the characteristic size of the inhomogeneities that we wish to highlight, in respect to near sight and far sight observations.

- $\quad \mathbf{t}_{1}$ and $\mathbf{t}_{2}$ : horizontal and vertical vectors used to compute the GLCM. These two directions have been selected because they coincide to the orientation of classical defects of most printing systems, because defaults often occur in the printing direction (vertical) and in the perpendicular direction of it.

\section{EXPERIMENTAL TESTING AND COMPARISON WITH PSYCHO-VISUAL ASSESSMENT}

In order to verify the relevance of the proposed score in respect to human observer evaluation, we carried out the following experiment:

Uniform color patches have been printed with various printers (laser, inkjet, retransfer) and on various supports (office paper, glossy photograph paper, APCO paper, white polymer). For the digitalization step, all samples are scanned at 2400 ppi in TIFF format, 24 bits, by the EPSON Scan Ver. 3.81 FR, 2002 driver, with a EPSON Perfection V700 PHOTO scanner. The "No color correction" option is selected. An overview of some samples is presented below. 


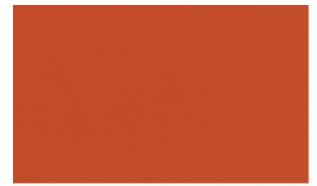

S_02

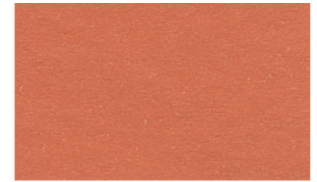

s 01

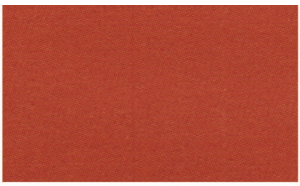

S_08

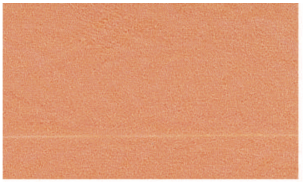

S 10

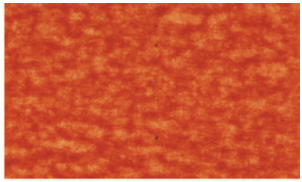

S 11

Figure 6. An overview of some scanned samples (in lower resolution than the original ones). s_02 is printed on glossy photograph paper, s_01 and s_08 on office paper, s_10 and s_11 on APCO paper.

The assessors were submitted to a ranking test, using an ordinale scale, unstructured, as it is presented by François Sauvageot in [14]. The assessors were presented randomly the samples to sort in respect to their perceived homogeneity, and were asked to place them on a one meter scale. The positions of the samples (in meter) are indicated by the dots in Figure 7, where one dot shape is attributed to each observer. Note that no rescaling of the positions has been performed: the dispersion of the positions for one sample partly comes from the different scaling adopted by the observers in their evaluation. The continuous line in Figure 7 represents the score computed with our method, represented by formula (15). We see that the scores follows fairly well the positioning of the samples by the observers on the metric scale.

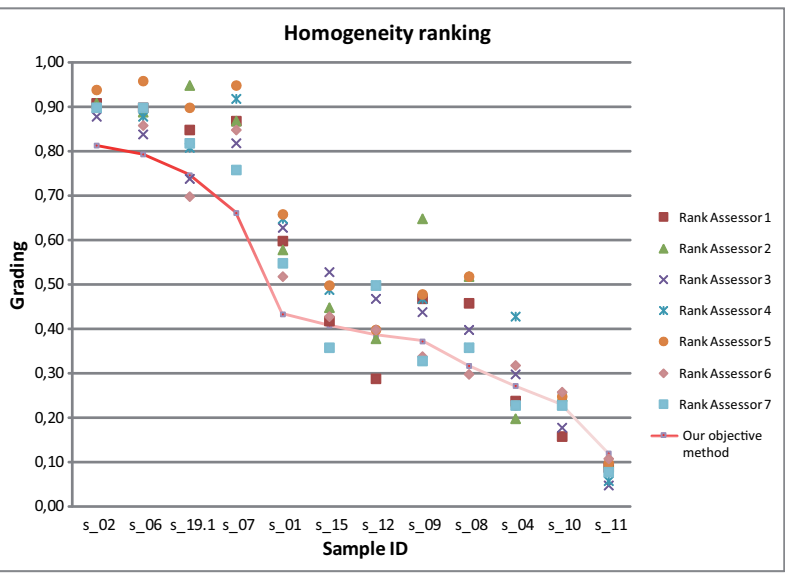

(a)

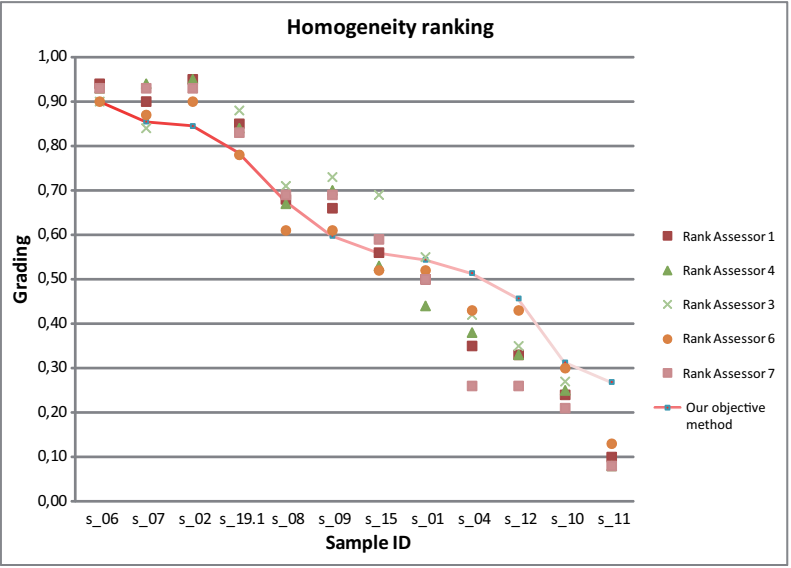

(c)

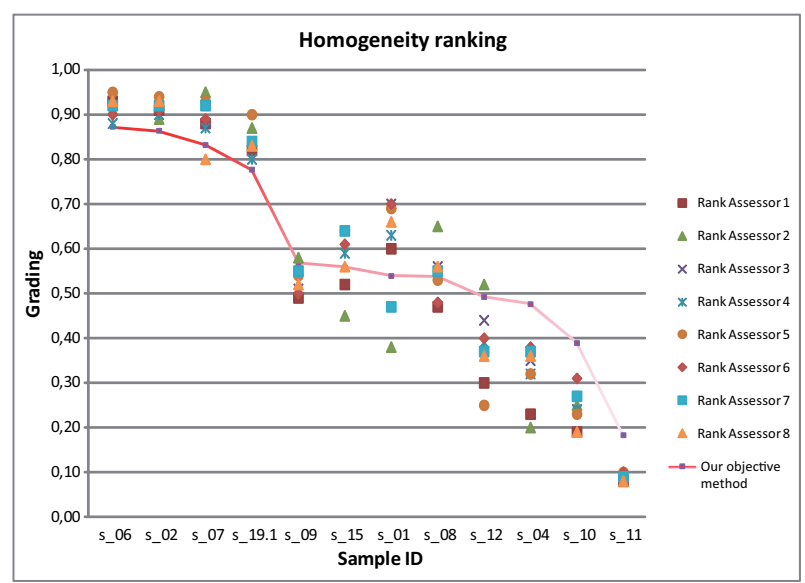

(b)

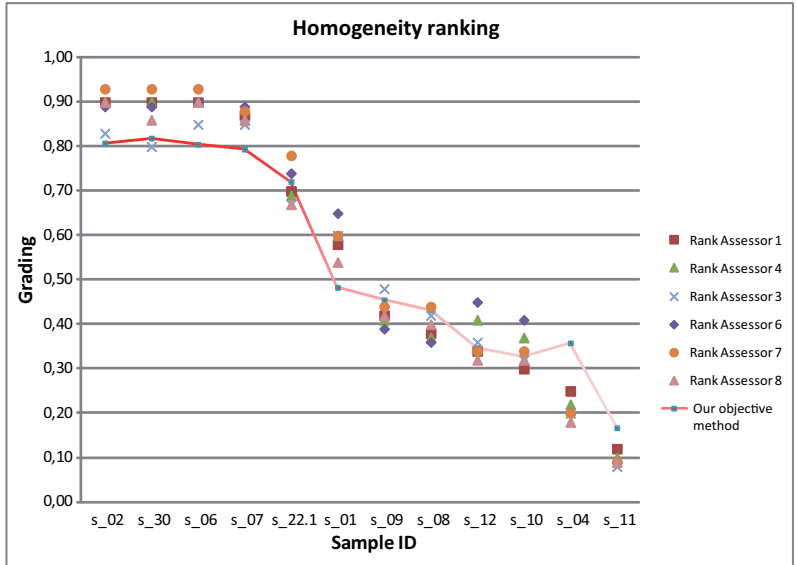

(d)

Figure 7. Comparison between objective and subjective evaluation for (a): a blue set of samples, (b): an orange set of samples, (c): a red set of samples, and (d): a gray set of samples. 
In order to assess the performance of our method, six different classes are defined by selecting six threshold values of the score $(0.8,0.7,0.6,0.52,0.3,0)$. Assessors scorings are also quantified into this six grades classification. The matching of the class given by observers and by our method is presented in Figure 8.

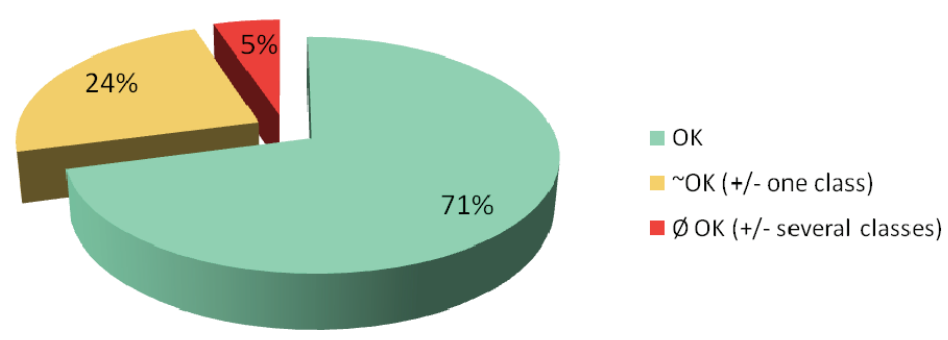

Figure 8. Percentage of samples classified in the same of six categories by assessors (subjective evaluation) and by our scoring method.

\section{CONCLUSION}

The index computed according to the method that we propose for the assessment of the homogeneity of printed colors is in accordance with the empirical assessment by a small panel of non-expert observers. In this sense, if the trend is confirmed for a larger set of observers, the algorithm that we have developed will appear consistent with the human visual perception. This homogeneity index is a first attribute which should be combined with other attributes in order to get a global quality score for printed natural images. It may also help to compare the performance of different printing systems in terms of visual quality.

\section{REFERENCES}

[1] H. Kipphan, [Handbook of Print Media] Springer Ed., (2001).

[2] M. Pedersen, N. Bonnier, J. Y. Hardeberg et al., "Attributes of a New Image Quality Model for Color Prints," Color and Imaging Conference, 2009(1), 204-209 (2009).

[3] M. Pedersen, and S. A. Amirshahi, "Framework for the Evaluation of Color Prints Using Image Quality Metrics," Conference on Colour in Graphics, Imaging, and Vision, 2010(1), 75-82 (2010).

[4] M. Gong, and M. Pedersen, "Spatial pooling for measuring color printing quality attributes," Journal of Visual Communication and Image Representation, 23(5), 685-696 (2012).

[5] Standard, [IEC 13660 Information technology-Office equipment-Measurement of image quality attributes for hardcopy output-Binary monochrome text and graphic images"-ISO] IEC, Genevas, Switzerland: International Organization for Standardization and International Electrotechnical Commission.(2001).

[6] A. Sadovnikov, P. Salmela, L. Lensu et al., [Mottling Assessment of Solid Printed Areas and Its Correlation to Perceived Uniformity] Springer Berlin Heidelberg, 42 (2005).

[7] M. Dube, F. Mairesse, J.-P. Boisvert et al., "Wavelet Analysis of Print Mottle," IEEE Transactions on Image Processing, (2005).

[8] R. M. Haralick, K. Shanmugam, and I. H. Dinstein, “Textural Features for Image Classification,” Systems, Man and Cybernetics, IEEE Transactions on, SMC-3(6), 610-621 (1973).

[9] G. Srinivasan, and G. Shobha, "Statistical texture analysis," proceedings of world academy of science, engg \& tech, 36, (2008).

[10] G. Sharma, [Digital Color Imaging Handbook] CRC Press, (2002).

[11] D. H. Pritchard, "U.S. Color Television Fundamentals: A Review," SMPTE Journal, 86(11), 819-828 (1977). 
[12] H. v. Helmholtz, and J. P. C. Southall, "Helmholtz's Treatise on Physiological Optics. Vol. II: The Sensations of Vision, trans. JPC Southall.(Translated from the Third German Edition)," The Optical Society of America, (1924), pp. 32-36.

[13] ITU, [Studio encoding parameters of digital television for standard 4:3 and wide-screen 16:9 aspect ratios], (2011).

[14] F. Depledt, [Evaluation sensorielle Manuel méthodologique] Tec Et Doc, (2009), pp. 130-132, in French. 